\title{
Control of a Stand-Alone Wound Rotor Synchronous Generator: Two Sliding Mode Approaches via Regulation of the d-Voltage Component
}

\author{
A. Dòria-Cerezo, V. I. Utkin, R. S. Muñoz-Aguilar, and E. Fossas
}

\begin{abstract}
In this brief two sliding mode control alternatives to regulate the stator voltage amplitude for a stand alone wound rotor synchronous generator are presented. Both controllers use the stator voltage $d$-component error in the sliding surface. In a first case an outer proportional-integral (PI) loop controller is added to provide the proper d-voltage component reference. The second approach consists in extending the dynamic system to include the integral term as state variable and to modify the former sliding surface by adding this new state. Finally, both simulations and experimental results validates the proposed algorithms.
\end{abstract}

Index Terms-Electrical machines, sliding mode control (SMC), stand-alone generation, stator voltage amplitude regulation, wound rotor synchronous generator (WRSM).

\section{INTRODUCTION}

$\mathbf{T}$ HE wound rotor synchronous machine (WRSM) is a doubly-fed electrical machine which can be fed simultaneously trough the stator and the rotor sides. This machine can be used for driving applications [1], [2], but its main application is as generator. Electrical energy is mainly generated interconnecting WRSM, setting up a theoretical infinite bus. Hence, this kind of machine is normally studied connected to an infinite bus called "power grid" [3]. When WRSM are directly connected to the power grid, the stator voltage and frequency are established by the power grid, while the rotor voltage helps to improve the power factor and to compensate the reactive power at the connection point.

In this brief the stand-alone generation is studied. This configuration, which is significantly different to the grid connected topology, is given in several emergency devices (as diesel generators) or hybrid electrical vehicles [4], and can be also applied to the large wind generators connected to the grid through

Manuscript received January 11, 2011; accepted April 03, 2011. Manuscript received in final form April 08, 2011. Recommended by Associate Editor J. Y. Lew. This work was supported in part by the Spanish Government Research Projects DPI2010-15110 (A. Dòria-Cerezo and E. Fossas), ENE2008-06841C02-01/ALT (R. S. Muñoz-Aguilar), and DPI2008-01408 (E. Fossas).

A. Dòria-Cerezo is with the Department of Electrical Engineering and the Institute of Industrial and Control Engineering, Universitat Politècnica de Catalunya, 08028 Barcelona, Spain (e-mail: arnau.doria@upc.edu).

V. I. Utkin is with the Department of Electrical Engineering, The Ohio State University, Columbus OH 43210 USA (e-mail: utkin@ece.osu.edu).

R. S. Muñoz-Aguilar is with the Department of Electrical Engineering, Universitat Politècnica de Catalunya, 08222 Terrassa, Spain (e-mail: raul.munozaguilar@upc.edu).

E. Fossas is with the Department of Automatic Control and the Institute of Industrial and Control Engineering, Universitat Politècnica de Catalunya, 08028 Barcelona, Spain (e-mail: enric.fossas@upc.edu).

Color versions of one or more of the figures in this brief are available online at http://ieeexplore.ieee.org.

Digital Object Identifier 10.1109/TCST.2011.2142310 a back-to-back converter [5], [6]. In this scenario neither amplitude nor frequency of stator voltage are fixed. For the standalone configuration, although the mechanical speed determines the frequency, the rotor voltage is used to set the stator amplitude.

Several examples can be found in the literature about the control of synchronous machines. The permanent magnet case (PMSM) is the most studied. However, examples for the WRSM are not so extensive. For motor purposes, decoupling methods are the most common [7], but recently nonlinear control techniques such as passivity-based control [1] or Lyapunov-based designs [8] are also used for driving applications. The case of a synchronous generator connected to the power grid is the most treated in the literature using, for example, advanced linear control techniques [9], sliding modes [10], or passivity-based control [11]. Linear PI controllers are the most used algorithms in the industry for the stand alone generation configuration. But there is a lack of references in the literature for this application, probably due to nonlinear nature of its output. Only few papers deal with this topology, as a preliminary sliding mode algorithm in [12] or a classical PI controller for a synchronous generator supplying an active de load [13].

The main contributions of this work are two different sliding mode control (SMC) algorithms for the stator voltage amplitude regulation of a wound rotor synchronous generator feeding an isolated resistive unknown load, under the assumption that the mechanical speed is externally regulated. In both cases, SMC is not directly applied to the system natural output (stator voltage amplitude), as suggested in [12]. Instead, the sliding surface takes into account the $d$-component of the stator voltage. The main difference between the two control laws lies in the sliding surface definition. As a first approach, two control loops are proposed: an inner SMC where the sliding surface is the error between the d component and a reference (presumed that all the coefficients are known) which results in a linear sliding dynamics plus a proportional-integral (PI) controller, to make the closed-loop system robust with respect to parameter variations. The second algorithm, so-called direct scheme, includes an integral term into the sliding surface that makes the system robust against parameter variation.

The SMC technique is particularly attractive for control industry devices due to its robustness and easy implementation. SMC can be found in several applications such as electropneumatic systems [14] or fuel cells [15]. This technique is specially appropriate for variable structure systems (VSS) as power converters [16]. Even that electrical machines are not VSS, SMC has been proposed for controlling them [17]. Mainly, this is because of the use of power converters applying the electrical machines control voltages, and the discrete values that these voltages take. 


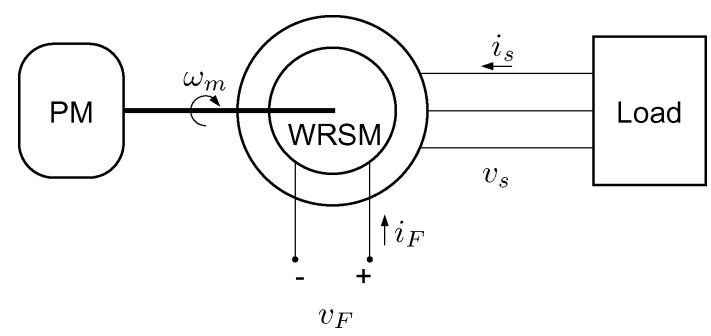

Fig. 1. Scheme of a stand-alone WRSG.

This brief is organized as follows. First, the system and its dynamics are introduced in Section II. In Section III the sliding mode controller with an external PI loop is designed. The use of a dynamic extension allows to design a different control law, where the integral term is directly considered in the sliding surface. This is presented in Section IV. Simulation and experimental results are shown in Sections V and VI, respectively, and, finally, conclusions are stated in Section VII.

\section{SySTEM DESCRIPTION}

Fig. 1 shows the proposed scenario: the primary mover drags a WRSM, which acts as a generator to feed an isolated load. $\omega_{m}$ is the mechanical speed, $v_{s}, v_{F}$ are the stator and field voltages, and $i_{s}, i_{F}$ are the stator and field currents.

As explained before, this system is different from the typical grid connection where the frequency and the voltage amplitudes are fixed by the grid. For an isolated connection the frequency is determined by the mechanical speed provided by the primary mover (PM), while the voltage amplitude must be assured by the rotor field voltage.

\section{A. Dynamic Model}

From the well-known dynamical equations (in the $d q$-coordinates) of the WRSM, and the interconnection rules with a pure resistor load, the whole dynamical system is presented. In this brief the machine is assumed to be symmetric (all windings are equal), and the stator-rotor cross inductances are considered smooth and sinusoidal functions of the rotor angle with just the fundamental term. Then, the $d q$ transformation allows to decouple one of the phases, to refer all variables to a common reference frame, and to obtain constitutive laws independent of the relative angle between rotor and stator [18]. Also, for the control design it is considered a constant mechanical speed even that in a real application it can be affected by load changes due to the magnetic coupling, specially when a sudden load is connected. Taking into account that mechanical speed is externally regulated by the primary mover, the electrical part of the WRSM with a resistive load can be written in an affine form as

$$
L \frac{d x}{d t}=A x+B v_{F}
$$

where

$$
L=\left(\begin{array}{ccc}
L_{s} & 0 & L_{m} \\
0 & L_{s} & 0 \\
L_{m} & 0 & L_{F}
\end{array}\right)
$$

is the inductance matrix

$$
A=\left(\begin{array}{ccc}
-\left(R_{s}+R_{L}\right) & \omega L_{s} & 0 \\
-\omega L_{s} & -\left(R_{s}+R_{L}\right) & -\omega L_{m} \\
0 & 0 & -R_{F}
\end{array}\right)
$$

and

$$
B=\left(\begin{array}{l}
0 \\
0 \\
1
\end{array}\right)
$$

State variables are the $d q$-stator and field currents $x^{T}=$ $\left(i_{d}, i_{q}, i_{F}\right) \in \mathbb{R}^{3}, R_{s}$ and $R_{F}$ are the stator and field resistances, $L_{s}, L_{m}$, and $L_{F}$ are the stator, mutual, and field inductances, $\omega$ is the electrical speed $\left(\omega=n_{p} \omega_{m}\right.$, where $n_{p}$ is the number of poles pairs), and $v_{F}$ is the field voltage which will be used as a control input. Moreover, the following parameter can be defined $\mu=L_{s} L_{F}-L_{m}>0$.

\section{B. Equilibrium Points}

The equilibrium points can be parametrized by the control input $v_{F}$, this resulting in $x^{*}=-A^{-1} B v_{F}$, which yields a straight line defined by

$$
x^{* T}\left(v_{F}\right)=\left[-\frac{\omega^{2} L_{s} L_{m}}{R_{F}\left|Z_{s}\right|^{2}},-\frac{\omega L_{m}\left(R_{s}+R_{L}\right)}{R_{F}\left|Z_{s}\right|^{2}}, \frac{1}{R_{F}}\right] v_{F}
$$

where $\left|Z_{s}\right|^{2}=\omega^{2} L_{s}^{2}+\left(R_{s}+R_{L}\right)^{2}$.

\section{Control Objective}

This machine must ensure stator voltage amplitude and frequency. For a synchronous machine, the stator frequency is directly given by the mechanical speed, which, in this brief is assumed to be constant and externally regulated. Then, the system output is the stator voltage amplitude $V_{s}$, which can be easily obtained, in a $d q$-framework as

$$
V_{s}=\sqrt{v_{d}^{2}+v_{q}^{2}}
$$

For a resistive load case

$$
\left(\begin{array}{l}
v_{d} \\
v_{q}
\end{array}\right)=R_{L}\left(\begin{array}{l}
i_{d} \\
i_{q}
\end{array}\right)
$$

and (3) can be expressed in current terms as

$$
V_{s}=R_{L} \sqrt{i_{d}^{2}+i_{q}^{2}} .
$$

Note that, a fix value of $V_{s}$ in (5), namely $V_{s}=V_{\text {ref }}$

$$
V_{\text {ref }}^{2}=R_{L}^{2}\left(i_{d}^{2}+i_{q}^{2}\right)
$$

implies that the control goal defines a cylinder in the state space, see Fig. 2.

Then, the desired equilibrium points are the intersection of the straight line (2) of the WRSM and the cylinder (6). Using polar coordinates

$$
i_{d}=I_{s} \cos \delta \quad i_{q}=I_{s} \sin \delta
$$

where $I_{s}=V_{s} / R_{L}$, it is easy to obtain

$$
i_{d}^{*}=\frac{V_{\text {ref }}}{R_{L}} \cos \delta^{*}
$$




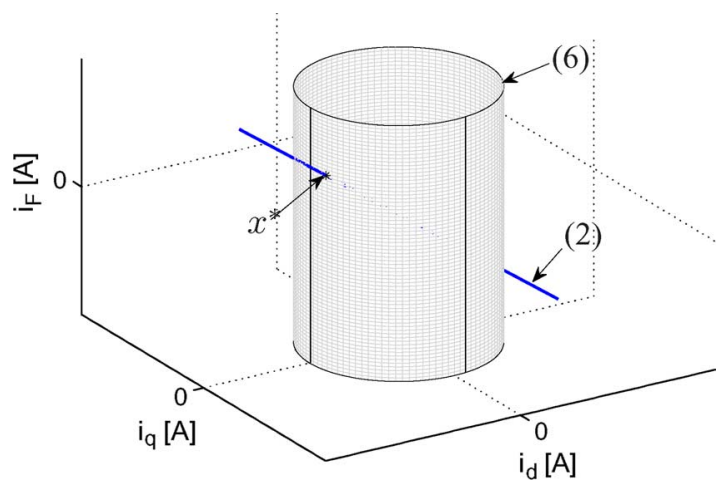

Fig. 2. Intersection of the control goals and the equilibrium points distribution of the system.

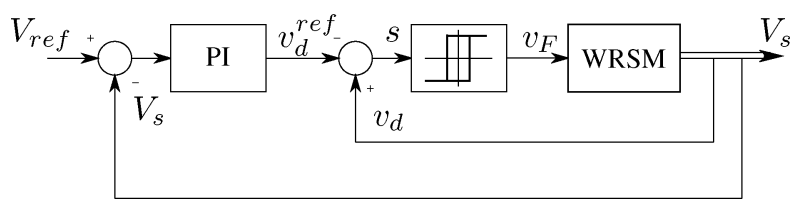

Fig. 3. SMC scheme for a WRSG.

$$
\begin{aligned}
i_{q}^{*} & =\frac{V_{\text {ref }}}{R_{L}} \sin \delta^{*} \\
i_{F}^{*} & =-\frac{V_{\text {ref }}}{R_{L}} \frac{L_{s}}{L_{m} \cos \delta^{*}}
\end{aligned}
$$

and, from (7), (8), and the equilibrium point given by the $i_{d}$ dynamics (first row of (1))

$$
\delta^{*}=\arctan \left(\frac{R_{s}+R_{L}}{\omega L_{s}}\right)
$$

In fact, there exist two solutions, given by the two values of the arctan function, which are depicted in Fig. 2.

Furthermore, the value of the field voltage in equilibria is

$$
v_{F}^{*}= \pm \frac{R_{F} L_{s}}{R_{L} L_{m} \cos \delta^{*}} V_{\text {ref }} .
$$

\section{SMC WITH AN OUTER-PI LOOP}

Fig. 3 shows the proposed control algorithm based on two loops; an inner-loop based on SMC and a PI outer loop which provides the stator voltage amplitude $d$-component reference, $v_{d}^{\text {ref }}$, to reach $V_{\text {ref }}$. This is an easy solution to simplify the sliding surface and to reduce its dependence only on $i_{d}$. Roughly speaking, when the system is faced with a perturbation, the PI loop places the sliding surface in the appropriate regulation point. This case differs from the one that will be presented in Section IV, where the integral term is considered as a new state. The whole stability proof is based on the assumption of a fast inner-loop, with respect to the PI dynamics.

\section{A. Inner-Loop: A Sliding Mode Controller}

The sliding mode controller enforces the system trajectories to reach and keep on the sliding surface

$$
s(x)=v_{d}(x)-v_{d}^{\mathrm{ref}}
$$

where $v_{d}^{\text {ref }}$ is defined by the outer-loop PI controller. From (4)

$$
s(x)=R_{L} i_{d}-v_{d}^{\mathrm{ref}} .
$$

Note that $s(x)=0$ defines the plane in $i_{d}=v_{d}^{\text {ref }} / R_{L}$. This case differs from [12], where the sliding surface was the cylinder (6). The equivalent control, $u_{\mathrm{eq}}$ is the solution of

$$
\frac{\partial s}{\partial x} L^{-1}\left(A x+B u_{\mathrm{eq}}\right)=0 .
$$

It results in

$$
u_{\mathrm{eq}}=\frac{1}{L_{m}}\left(-L_{F}\left(R_{s}+R_{L}\right) i_{d}+\omega L_{s} L_{F} i_{q}+L_{m} R_{F} i_{F}\right) .
$$

There is sliding motion on $s(x)=0$ provided that the reachability condition $s \cdot(d s / d t)<0$ holds. From (1)

$$
s \cdot \frac{d s}{d t}=s \frac{\partial s}{\partial x} L^{-1}\left(A x+B v_{F}\right)<0
$$

that, using (1) and (10), can be written as

$$
-s \frac{R_{L} L_{m}}{\mu}\left(v_{F}-u_{\text {eq }}\right)<0
$$

finally, as $R_{L}, L_{m}, \mu>0$, the reachability condition yields

$$
s\left(u_{\mathrm{eq}}-v_{F}\right)<0 .
$$

Using a bang-bang rotor voltage $\left(v_{F}= \pm V_{\mathrm{DC}}\right)$, the following switching policy:

$$
v_{F}= \begin{cases}V_{\mathrm{DC}}, & \text { if } s>0 \\ -V_{\mathrm{DC}}, & \text { if } s<0\end{cases}
$$

fulfils the reachability condition presumed that $-V_{\mathrm{DC}}<u_{\mathrm{eq}}<$ $V_{\mathrm{DC}}$.

The remaining dynamics (ISD) results in the following linear system:

$$
\begin{aligned}
\frac{d i_{q}}{d t} & =-\frac{R_{s}+R_{L}}{L_{s}} i_{q}-\frac{\omega L_{m}}{L_{s}} i_{F}-\frac{\omega}{R_{L}} v_{d}^{\mathrm{ref}} \\
\frac{d i_{F}}{d t} & =\frac{\omega L_{s}}{L_{m}} i_{q}-\frac{R_{s}+R_{L}}{L_{m} R_{L}} v_{d}^{\mathrm{ref}}
\end{aligned}
$$

which is stable since $\left(R_{s}+R_{L}\right) / 2 \omega L_{s}<1$.

\section{B. Outer-Loop: A PI Controller}

The outer-loop consists in a simple PI controller. Considering a fast inner-loop, the closed-loop system, (1) and (11), reduces to (12) and (13), where the new input is $v_{d}^{\text {ref }}$ and the output still is $V_{s}$. Though the output remains nonlinear, presuming the ideal sliding dynamics achieves the steady-state, (3) can be rewritten as

$$
V_{s}=\sqrt{\left(v_{d}^{\text {ref }}\right)^{2}+R_{L}^{2} i_{q}^{2}}
$$

linearizing around $\left(v_{d}^{r e f *}, i_{q}^{*}\right)$ such that

$$
V_{\mathrm{ref}}=\sqrt{\left(v_{d}^{r e f *}\right)^{2}+R_{L}^{2} i_{q}^{* 2}} .
$$

Equation (14) results in 


$$
V_{s} \simeq V_{\mathrm{ref}}+\frac{v_{d}^{r e f *}}{V_{\mathrm{ref}}}\left(v_{d}^{\mathrm{ref}}-v_{d}^{r e f *}\right)+\frac{R_{L}^{2} i_{q}^{*}}{V_{\mathrm{ref}}}\left(i_{q}-i_{q}^{*}\right)
$$

where

$$
v_{d}^{\mathrm{ref} *}=V_{\mathrm{ref}} \cos \delta^{*}
$$

Using (8), (15), and (16), the nonlinear equation (14) can be approximated by

$$
V_{s} \simeq v_{d}^{\mathrm{ref}} \cos \delta^{*}+R_{L} i_{q} \sin \delta^{*}
$$

From (12), (13), and (17) the transfer function $V_{s} / v_{d}^{\text {ref }}$ is obtained. Namely ${ }^{1}$

$$
G(s)=\frac{\cos ^{2} \delta^{*} s^{2}+\omega^{2}}{\cos \delta^{*} s^{2}+\omega \sin \delta^{*} s+\omega^{2} \cos \delta^{*}} .
$$

The closed-loop system transfer function is given by

$$
W(s)=\frac{c_{3} s^{3}+c_{2} s^{2}+c_{1} s+c_{0}}{b_{3} s^{3}+b_{2} s^{2}+b_{1} s+b_{0}}
$$

where $c_{3}=k_{p} \cos ^{2} \delta^{*}, c_{2}=k_{i} \cos ^{2} \delta^{*}, c_{1}=k_{p} \omega^{2}, c_{0}=k_{i} \omega^{2}$ and

$$
\begin{aligned}
& b_{3}=\cos \delta^{*}\left(k_{p} \cos \delta^{*}+1\right) \\
& b_{2}=\left(\omega \sin \delta^{*}+k_{i} \cos ^{2} \delta^{*}\right) \\
& b_{1}=\omega^{2}\left(k_{p}+\cos \delta^{*}\right) \\
& b_{0}=k_{i} \omega^{2} .
\end{aligned}
$$

Application of the Routh-Hurwitz criterion yields to the next stability conditions

$$
\begin{aligned}
k_{p} & >-\frac{1}{\cos \delta^{*}} \\
k_{i} & >0 \\
k_{i} & <\frac{\omega\left(k_{p} \sin \delta^{*}+\cos \delta^{*}\right)}{\cos \delta^{*} \sin \delta^{*}} .
\end{aligned}
$$

\section{Direct SLiding Mode Controller}

In this section, a direct sliding mode controller is presented. The sliding surface is still based on the error of the $d$-component of the stator voltage, and an integral action is added in order to robustify the controller [19]. This dynamic extension is also used to reduce the chattering effects, see [17] for details. It is worth to mention that there is not possible to add a proportional component of the error in terms of $V_{s}$. As proposed in [12], this choice implies into a failure of the transversality condition. Fig. 4 shows the proposed control scheme.

\footnotetext{
${ }^{1}$ This transfer function takes the form $G(s)=1+Q(s)$. Hence, a jump in $v_{d}^{\text {ref }}$ results in a jump in a $V_{s}$. But this only occurs presuming ideal sliding dynamics. In a real application, $V_{\mathrm{DC}}$ limitations and the own dynamics of the inner loop will filter the output signal $V_{s}$.
}

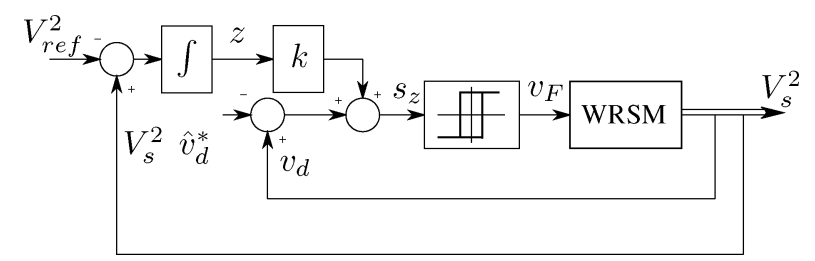

Fig. 4. Direct SMC scheme for a wound rotor synchronous generator.

\section{A. Control Design}

The integral term entails the extension of the system with a new variable $z$ defined by

$$
\frac{d z}{d t}=V_{s}^{2}-V_{\mathrm{ref}}^{2}
$$

The new sliding surface $s_{z}$ is

$$
s_{z}(x, z)=v_{d}(x)-\hat{v}_{d}^{*}+k z
$$

where $\hat{v}_{d}^{*}$ is the $v_{d}$ nominal value given $V_{\text {ref }} \cdot \hat{v}_{d}^{*}$ is obtained from (7) and (4) using the nominal plant parameters ${ }^{2}$

$$
v_{d}^{*}=V_{\text {ref }} \cos \hat{\delta}^{*} .
$$

The equivalent control is obtained and it results in

$$
u_{\mathrm{zeq}}=u_{\mathrm{eq}}+\frac{\mu}{L_{m} R_{L}} k \dot{z}
$$

equals to the former equivalent control plus the derivative of the new integral term.

Now, the control law is designed with the extended system

$$
L_{z} \frac{d z_{e}}{d t}=A_{z}\left(z_{e}\right)+B_{z} v_{F}
$$

where $z_{e}^{T}=\left[i_{d}, i_{q}, i_{F}, z\right]$

$$
\begin{aligned}
L_{z} & =\left(\begin{array}{cccc}
L_{s} & 0 & L_{m} & 0 \\
0 & L_{s} & 0 & 0 \\
L_{m} & 0 & L_{F} & 0 \\
0 & 0 & 0 & 1
\end{array}\right) \\
A_{z}\left(z_{e}\right) & =\left(\begin{array}{c}
A x \\
R_{L}^{2}\left(i_{d}^{2}+i_{q}^{2}\right)-V_{\mathrm{ref}}^{2}
\end{array}\right)
\end{aligned}
$$

and $B_{z}^{T}=(0,0,1,0)$. Taking into account that $R_{L}, L_{m}, \mu>0$ and proceeding as usual, reachability condition yields

$$
s_{z}\left(u_{z e q}-v_{F}\right)<0
$$

and, consequently, the switching control policy

$$
v_{F}= \begin{cases}V_{\mathrm{DC}}, & \text { if } s_{z}>0 \\ -V_{\mathrm{DC}}, & \text { if } s_{z}<0\end{cases}
$$

guaranties sliding motion on $s_{z}=0$ presumed that $-V_{\mathrm{DC}}<$ $u_{\text {zeq }}<V_{\mathrm{DC}}$.

${ }^{2}$ The hat in $\hat{v}_{d}^{*}$ and $\hat{\delta}^{*}$ denotes that this value is estimated depending on the nominal parameters of $R_{L}, R_{s}$ and $L_{s}$. 


\section{B. Ideal Sliding Dynamics}

The controller defined in (20) ensures sliding modes on the switching surface. Using $s_{z}(x, z)=0$ and $v_{F}=u_{\text {zeq }}$ in (19), and defining a new variable $\xi=L_{s} i_{d}+L_{m} i_{F}$, the ideal sliding dynamics, in terms of the error, $e^{T}=\left(i_{d}-i_{d}^{*}, i_{q}-i_{q}^{*}, \xi-\xi^{*}\right)$, can be written as

$$
\frac{d e}{d t}=A_{\operatorname{lin}} e-k R_{L} g(e)
$$

where

$$
A_{\text {lin }}=\left(\begin{array}{ccc}
-2 k R_{L} i_{d}^{*} & -2 k R_{L} i_{q}^{*} & 0 \\
0 & -\frac{R_{s}+R_{L}}{L_{s}} & -\frac{\omega}{L_{s}} \\
-\left(R_{s}+R_{L}\right) & \omega L_{s} & 0
\end{array}\right)
$$

represents the linear part, and

$$
g(e)^{T}=\left(e_{1}^{2}+e_{2}^{2}, 0,0\right)
$$

include the nonlinearities. Local stability is given by $k>0$ if $i_{d}^{*}>0$, which can be proved applying the Routh-Hurwitz criteria to the characteristic polynomial of (22). ${ }^{3}$

Local stability claimed above guarantees the existence of $P=P^{T}>0$ and $Q=Q^{T}>0$ (see [20, Th. 4.6]) so that

$$
e^{T}\left(A_{\operatorname{lin}}^{T} P+P A_{\operatorname{lin}}\right) e=-e^{T} Q e .
$$

Let us take $V(e)=e^{T} P e$, then

$$
\begin{aligned}
\frac{d V}{d t} & =e^{T}\left(A_{\text {lin }}^{T} P+P A_{\text {lin }}\right) e-2 k R_{L} e^{T} P g(e) \\
& \leq-\lambda_{Q}\|e\|^{2}-2 k R_{L}\left(e_{1} p_{11}+e_{2} p_{22}+e_{3} p_{33}\right)\|e\|^{2}
\end{aligned}
$$

where $\lambda_{Q}$ is the minimum eigenvalue $Q$, and $p_{11}, p_{12}, p_{13}$ are the terms in the first column of $P$. This concludes that stability of (21) holds in the region defined by

$$
\left|e_{1} p_{11}+e_{2} p_{22}+e_{3} p_{33}\right|<\frac{\lambda_{Q}}{2|k| R_{L}} .
$$

Notice that the stability is only guaranteed for a region in the state space. This result is not surprising and is related to the existence of two different equilibrium points as is shown in Fig. 2.

\section{Simulations}

In this Section the simulation results using the designed controller are presented. The used WRSM is a 4 poles, $2.4 \mathrm{~kW}$ machine with the following parameters: $R_{s}=3.06 \Omega, L_{s}=0.48$ $\mathrm{H}, L_{m}=0.31 \mathrm{H}, R_{F}=2.48 \Omega, L_{F}=0.24 \mathrm{H}$. Mechanical speed is set to $\omega_{m}=1500 \mathrm{rpm}$ and $V_{\mathrm{DC}}=35 \mathrm{~V}$. Initial conditions are $V_{\text {ref }}=200 \sqrt{2} \mathrm{~V}$ with a resistive load $R_{L}=120 \Omega$. Simulation test shows the response of the closed loop system under a load change to $R_{L}=64 \Omega$ at $t=0.05 \mathrm{~s}$. The controller gains are: $k_{p}=1000, k_{i}=100$ (for the PI controller) and $k=0.2$ (for the direct scheme). Simulations have been

\footnotetext{
${ }^{3} \mathrm{As}$ pointed out at the end of Section II, two equilibria are possible. To reach the value corresponding to $\delta^{*}=\arctan \left(\left(R_{s}+R_{L}\right) / \omega L_{s}\right)+\pi$, i.e., $i_{d}^{*}<0$, local stability condition also holds with $k<0$.
}
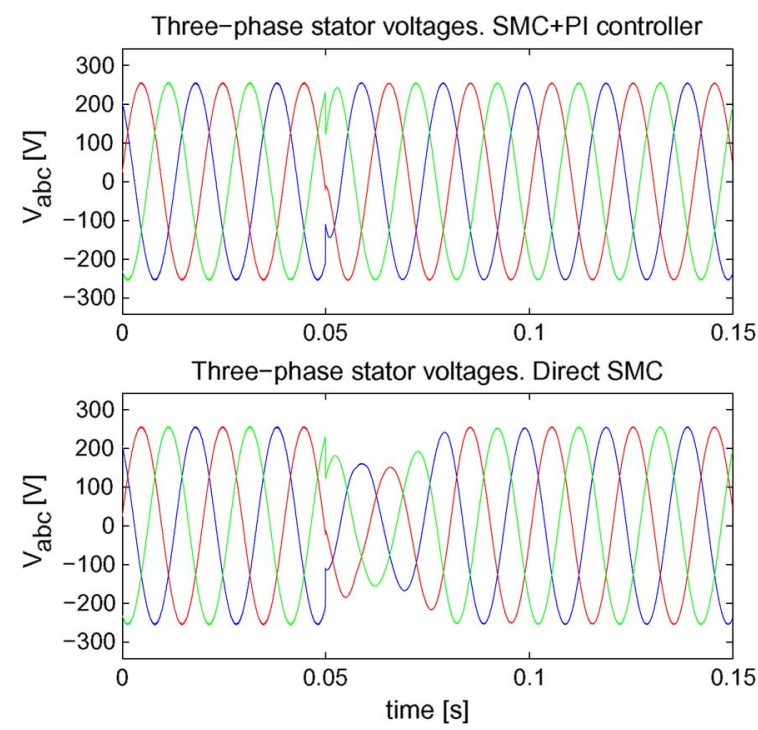

Fig. 5. Simulation results: Three phase stator voltages.
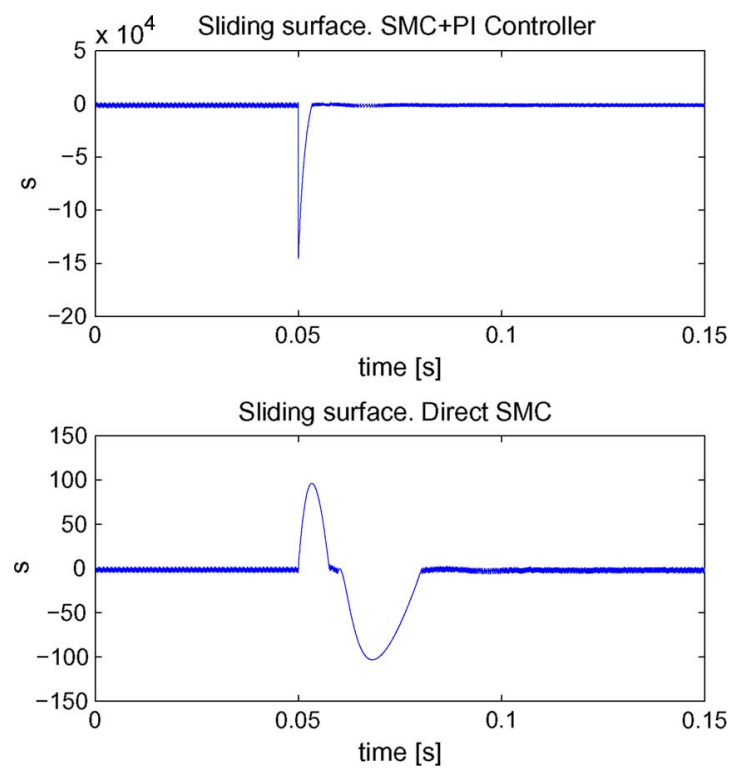

Fig. 6. Simulation results: sliding surfaces.

performed using a variable step integration method, with a zero order hold with $10 \mathrm{kHz}$ of sampling frequency.

Fig. 5 shows the three phase stator voltages. In both cases, regulation of the stator voltage amplitude is achieved. The fast response of the first controller algorithm is thanks to the proportional action of the PI loop, which, as has been pointed out in Section IV, could not be included in the direct scheme to hold the transversality condition.

Fig. 6 shows the sliding surfaces. Note that in the second case, sliding is lost twice for a short time after the load change. The first time is due to the sudden load variation, while the second time is caused by the equivalent control (18) which is out of range $\left(\left|u_{z e q}\right|>V_{\mathrm{DC}}\right)$. See also Fig. 7 , where the field voltage 

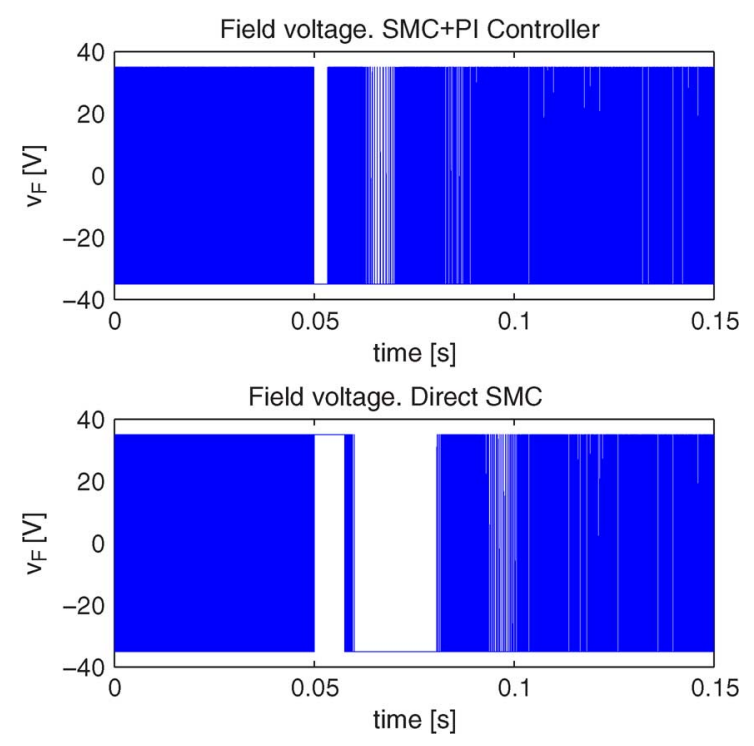

Fig. 7. Simulation results: field voltages.

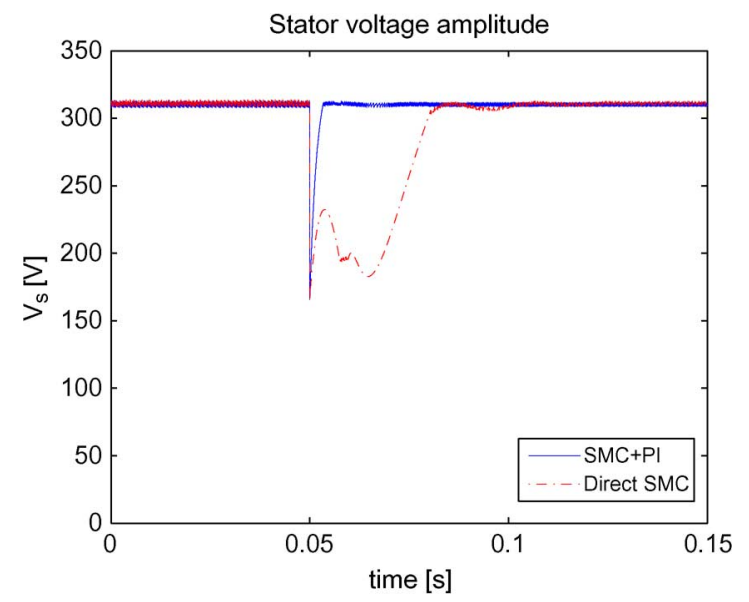

Fig. 8. Simulation results: stator voltage amplitudes.

$v_{F}$ is shown. Remark the long time the control variable remains saturated compared with the SMC+PI control law.

Velocity response can be observed in Figs. 8 and 9, where stator voltage amplitude and stator and field currents are displayed. The faster response of the SMC+PI controller, in front of the direct scheme, is clear.

\section{EXPERIMENTAL RESULTS}

\section{A. Hardware Description and Control Implementation}

The proposed controllers have been tested in a real plant. The WRSM is a $2.4 \mathrm{kVA}, 4$ poles three-phase machine. The nominal characteristics and the WRSM parameters are shown in Tables I and II, ${ }^{4}$ respectively. In this case, the WRSM is dragged by a DC motor which emulates the primary mover proposed in Section II. This machine, used to provide a constant speed of $1500 \mathrm{rpm}$ to the WRSM (which corresponds to $50 \mathrm{~Hz}$ frequency), is a 3

\footnotetext{
${ }^{4}$ The apostrophe signal indicates that the parameters are referred from the rotor to the stator, and $n$ is the transformation relationship.
}
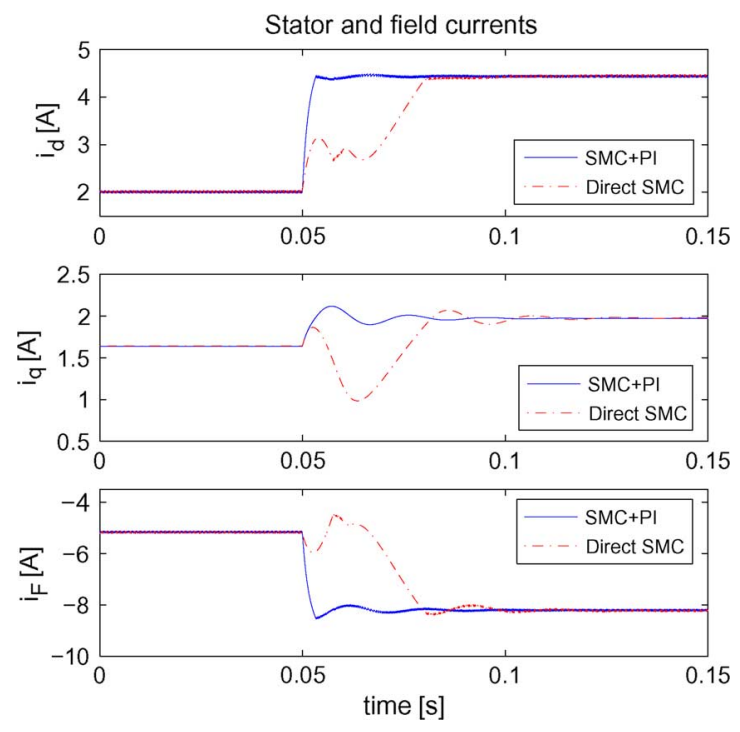

Fig. 9. Simulation results: stator and field currents.

TABLE I

WRSM CHARACTERISTICS

\begin{tabular}{|c|c|c|}
\hline$f=50 \mathrm{~Hz}$ & $n=1500 \mathrm{rpm}$ & $P=2.4 \mathrm{kVA}$ \\
\hline $3 \mathrm{ph}$ & $\Delta / \mathrm{Y}$ & $V_{F}=100 \mathrm{~V}$ \\
\hline$I_{F}=2.4 \mathrm{~A}$ & $V_{s}=220 / 380 \mathrm{~V}$ & $I_{s}=6.3 / 3.65 \mathrm{~A}$ \\
\hline
\end{tabular}

TABLE II

WRSM PARAMETERS

\begin{tabular}{|c|c|c|}
\hline$R_{s}=3.06 \Omega$ & $L_{s}=0.48 \mathrm{H}$ & $R_{F}=39.65 \Omega$ \\
\hline$L_{m}=0.31 \mathrm{H}$ & $L_{F}=3.87 \mathrm{H}$ & $n=4$ \\
\hline$R_{F}^{\prime}=2.48 \Omega$ & $L_{F}^{\prime}=0.24 \mathrm{H}$ & \\
\hline
\end{tabular}

$\mathrm{kW}$ machine with the $4 \mathrm{Q} 2$ commercial speed controller from Control Techniques Drives Ltd.

The power converter connected to the field circuit, is a full bridge DC/DC converter, which can provide $\pm V_{\mathrm{DC}}$ voltages. The $V_{\mathrm{DC}}$ voltage is obtained from the power grid with a diode rectifier, a $\mathrm{L}$ filter and a capacitor DC bus. For the experimental tests the bus voltage is set to $V_{\mathrm{DC}}=137.5 \mathrm{~V}$. The resistive load is composed of two interconnected banks, with a half or full load values (equivalent to $R_{L}=128 \Omega$ and $R_{L}=64 \Omega$ for the nominal voltage).

The control algorithm is programmed into a Texas Instruments floating point $150 \mathrm{MHz}$ Digital Signal Processor (DSP TMS 320F28335). The DSP has 16 ADC channels with 12-bit resolution, with a maximum conversion speed of 12.5 MSPS, 6 PWM, and 6 HRPWM outputs and 88 GPIO pins which can be used for communication purposes.

By using two differential sensors, and assuming a balanced load, three phase stator voltages are measured. Position is measured as well, in order to compute the dq transformation. These measures are acquired by the DSP which is programmed from a personal computer. Real Time Workshop C code generation from MATLAB/Simulink is used in order to simplify the code implementation to the DSP without using directly a $C$ code editor. Also Texas Target support package is used to configure the ADC, PWM, SPI, GPIO ports and interruptions. 

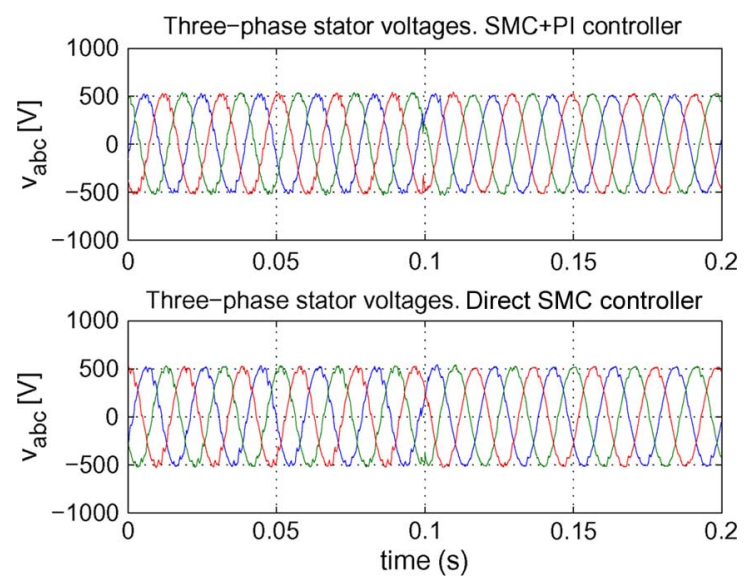

Fig. 10. Experimental results: three-phase stator voltages for a change from one half to full load value.

The control algorithms are coded in the Simulink environment and using the specific blocks developed for the TMSF28000 family of the DSP cards to implement dq-transformation, PI controllers and sign function. The sample time is fixed to $10^{-4} \mathrm{~s}$, which corresponds to $10 \mathrm{kHz}$ as a maximum frequency according to the DC/DC power converter features.

\section{B. Experimental Results}

Along these lines, experiments using the controllers designed in Sections III and IV are presented. The mechanical speed is fixed by the DC motor at $1500 \mathrm{rpm}$ (which corresponds to 50 $\mathrm{Hz}$ stator frequency).

The first experiment consists in validating the designed controllers under a load change. The reference line voltage is set to $380 \mathrm{Vrms}$ (which corresponds to $V_{\text {ref }}=220 \sqrt{2} \mathrm{~V}$ ), and the load is suddenly changed from the half to the full value. Figures from 10-12 show the three-phase stator voltages, the sliding surface and the switching control policy for each control scheme. As expected, both controllers regulate the stator voltage amplitude under a load change, Fig. 10. The switching function $s(x)$ is depicted in Fig. 11. Is worth to notice the difference of the switching function behavior between the two proposed algorithms due to the absence of a proportional gain in the Direct SMC scheme. Finally, the applied $v_{F}$ voltages for both control algorithms are shown in Fig. 12.

The second experiment consists in a stator voltage amplitude reference change. From an initial value of $250 \mathrm{Vrms}$ the reference is set to $380 \mathrm{Vrms}$, line values. In this case the load keeps to its half value $\left(R_{L}=128 \Omega\right)$. Figures from 13-15 show the three-phase stator voltages response, the switching control policy and the applied rotor field voltage, in front of a reference change. This experiment confirms that the SMC+PI algorithm regulates slightly faster than Direct SMC, Fig. 13. It can be appreciate that in steady-state, the Direct SMC algorithm contains undesired oscillations, due to the absence of a proportional term in the $V_{s}$ error. Note that, in Fig. 14, the sliding surface of the Direct SMC scheme remains close to zero. Again, this is because the direct scheme does not contain any proportional term.

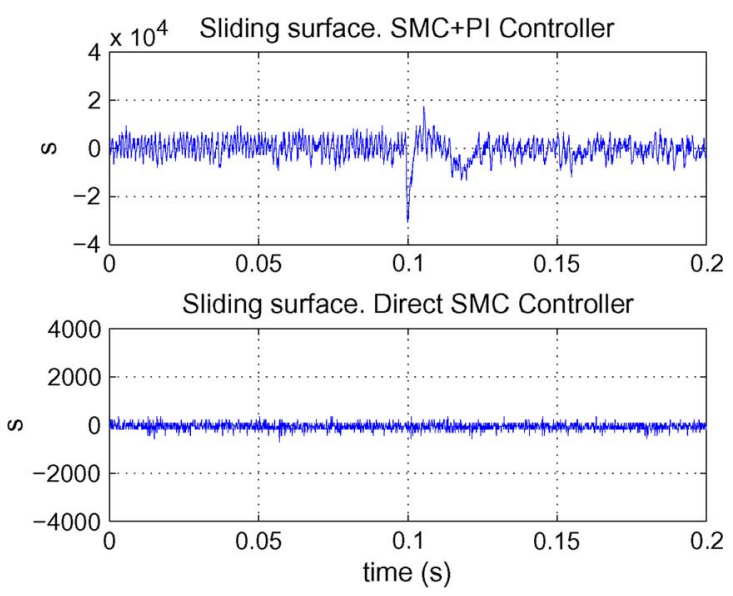

Fig. 11. Experimental results: sliding surface for a change from one half to full load value.
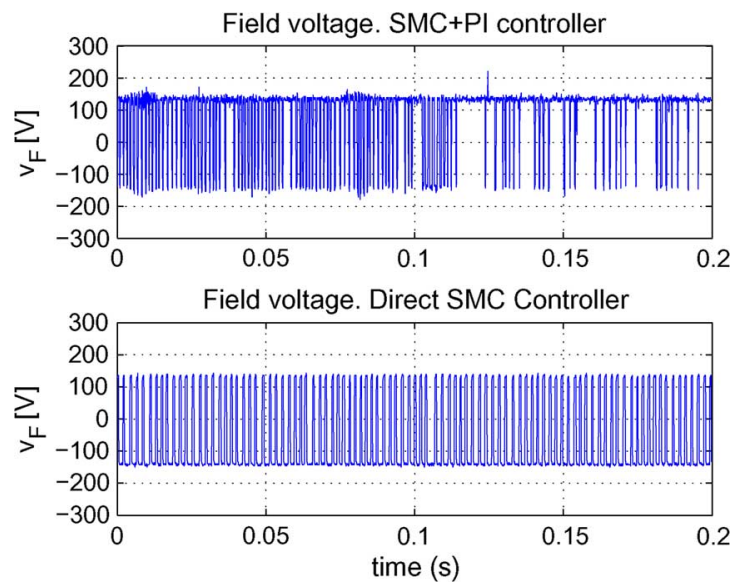

Fig. 12. Experimental results: switching control police for a change from half to full load value.
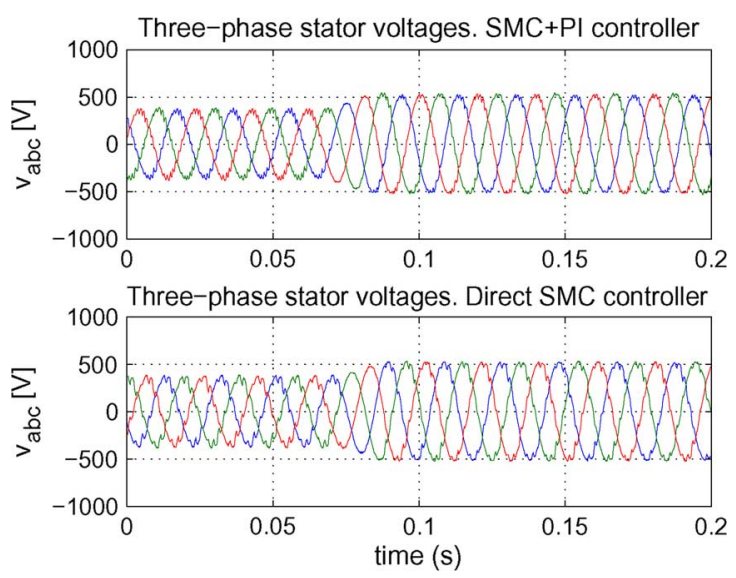

Fig. 13. Experimental results: three-phase stator voltages for a reference change from 250 to 380 with half load value.

\section{CONCLUSION}

In this brief two approaches for controlling the stator voltage amplitude of an isolated wound rotor synchronous generator feeding a resistive load are presented. Both controllers are designed using sliding modes, and taking the error of the $d$-voltage component as a sliding surface. The first controller is composed 

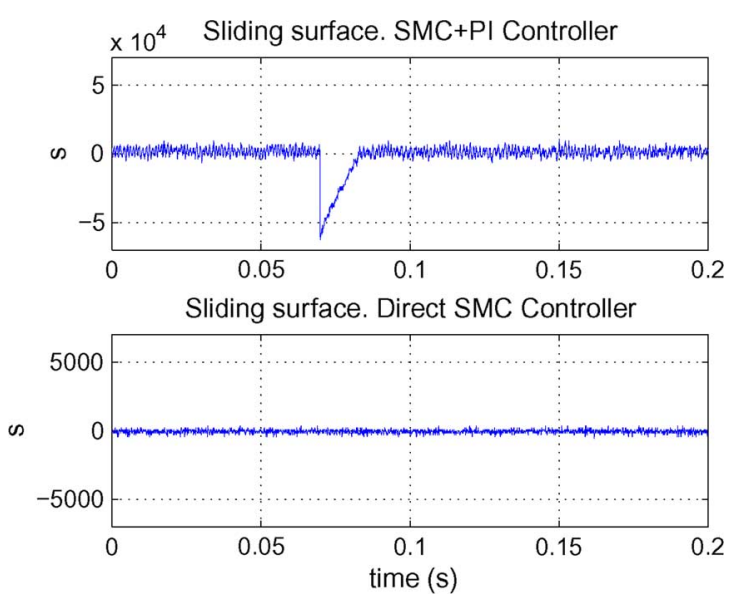

Fig. 14. Experimental results: sliding surface for a reference change from 250 to 380 with half load value.
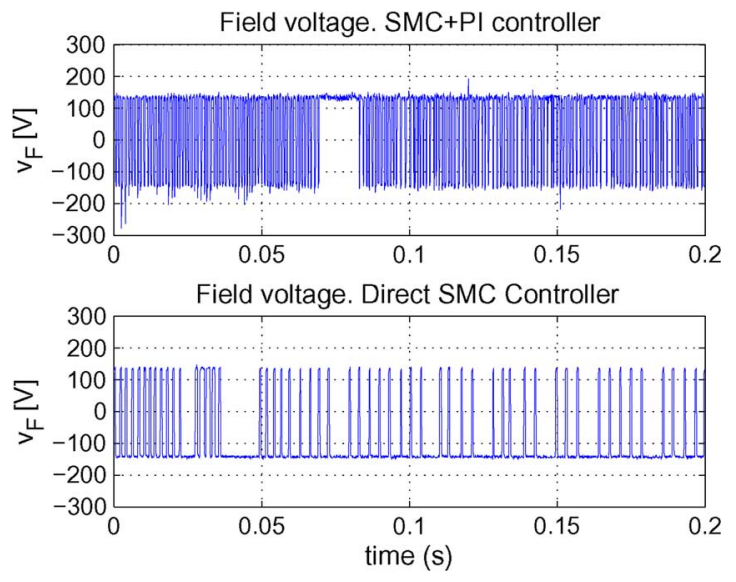

Fig. 15. Experimental results: switching control police for a reference change from 250 to 380 with half load value.

by an inner sliding mode-based loop, and a simple outer-loop PI controller, while the second approach suggest the use of a dynamic extension in the sliding surface.

Stability of both alternatives are studied. The inner SMC loop is globally stable, while the design of the outer PI loop is based on the linearization of the ideal sliding dynamics. The stability of the second control algorithm is first locally discussed, and a region of the space state is proved to be stable depending on the values of the controller gain.

The simulation results shows that the two possibilities achieve the control goal. However, since the direct scheme does not contain any proportional gain in $V_{s}-V_{\text {ref }}$, step changes in $V_{\text {ref }}$ only appear in the switching surface trough the integral term. On the contrary, the proportional part in the SMC+PI allows reflecting reference step changes in $s$ directly. The inclusion of the proportional part in the direct SMC implies the transversality condition fails at $i_{d}=0$, see [12].
Experimental tests, which have been also done for both controllers, validate the presented algorithms. The main lack of the second approach (the direct SMC) is the absence of a proportional gain on the output error, which implies a weaker performance of the controller.

\section{REFERENCES}

[1] A. Dòria-Cerezo, C. Batlle, and G. Espinosa, "Passivity-based control of a wound-rotor synchronous motor," IET Control Theory Appl., vol. 4, no. 10, pp. 2049-2057, 2010.

[2] C. Rossi, D. Casadei, A. Pilati, and M. Marano, "Wound rotor salient pole synchronous machine drive for electric traction," in Proc. IEEE Ind. Appl. Conf., 2006, pp. 1235-1241.

[3] P. M. Anderson and A. A. Fouad, Power System Control and Stability. Ames, IA: The Iowa State University Press, 1977.

[4] R. S. Munoz-Aguilar, A. Dòria-Cerezo, and P. F. Puleston, "Energy-based modelling and simulation of a series hybrid electric vehicle propulsion system," in Proc. 13th Euro. Conf. Power Electron. Appl., 2009, pp. 1-10.

[5] E. Torres and M. Garcia-Sanz, "Experimental results of the variable speed, direct drive multipole synchronous wind turbine twt 1650," Wind Energy, vol. 7, pp. 109-118, 2004.

[6] L. H. Hansen, L. Helle, F. Blaabjerg, E. Ritchie, S. Munk-Nielsen, H. Bindner, P. Sorensen, and B. Bak-Jensen, "Conceptual survey of generators and power electronics for wind turbines," Riso National Laboratory, Roskilde, Denmark, 2001.

[7] J. Chiasson, Modeling and High Performance Control of Electric Machines. New York: Wiley, 2005.

[8] A. E. Magri, F. Giri, A. Abouloifa, and M. Haloua, "Nonlinear control of wound-rotor synchronous-motor," in Proc. IEEE Int. Conf. Control Appl., 2006, pp. 3110-3115.

[9] K. T. Law, D. J. Hill, and N. R. Godfrey, "Robust controller structure for coordinated power system voltage regulator and stabilizer design," IEEE Trans. Control Syst. Technol., vol. 2, no. 3, pp. 220-232, May 1994.

[10] J. Cabrera-Vázquez, A. G. Loukianov, J. M. Canedo, and V. I. Utkin, "Robust controller for synchronous generator with local load via vsc," Elect. Power Energy Syst., vol. 29, pp. 348-359, 2007.

[11] R. Ortega, M. Galaz, A. Astolfi, Y. Sun, and T. Shen, "Transient stabilization of multimachine power systems with nontrivial transfer conductances," IEEE Trans. Autom. Control, vol. 50, no. 1, pp. 60-75, Jan. 2005.

[12] R. S. Munoz-Aguilar, A. Dòria-Cerezo, E. Fossas, and R. Cardoner, "Sliding mode control of a stand-alone wound rotor synchronous generator," IEEE Trans. Ind. Electron., 10.1109/TIE.2011.2116754.

[13] I. Jadric, D. Borojevic, and M. Jadric, "Modeling and control of a synchronous generator with an active dc loac," IEEE Trans. Power Electron., vol. 15, no. 2, pp. 303-311, Mar. 2000.

[14] M. Smaoui, X. Brun, and D. Thomasset, "Systematic control of an electropneumatic system: Integrator backstepping and sliding mode control," IEEE Trans. Control Syst. Technol., vol. 14, no. 5, pp. 905-913, Sep. 2006.

[15] C. Kunusch, P. F. Puleston, M. A. Mayosky, and J. Riera, "Sliding mode strategy for pem fuel cells stacks breathing control using a supertwisting algorithm," IEEE Trans. Control Syst. Technol., vol. 17, no. 1, pp. 167-174, Jan. 2009.

[16] D. Biel and E. Fossas, "SMC application in power electronics," in Variable Structure Systems: From Principles to Implementation, ser. IEE Control Series, A. Sabanovic, L. M. Fridman, and S. Spurgeon, Eds. London, U.K.: The Institution of Engineering and Technology, 2004, vol. 66, pp. 265-284.

[17] V. Utkin, J. Guldner, and J. Shi, Sliding Mode Control in Electromechanical Systems. New York: Taylor \& Francis, 1999.

[18] P. C. Krause, O. Wasynczuk, and S. D. Sudhoff, Analysis of Electric Machinery and Drive Systems. New York: Wiley, 2002.

[19] C. Edwards and S. K. Spurgeon, Sliding Mode Control. Theory and Applications. New York: Taylor \& Francis, 1998.

[20] H. K. Khalil, Nonlinear Systems, 3rd ed. Englewood Cliffs, NJ: Prentice-Hall, 2002. 